\title{
Über den Temperaturkoeffizienten der mole- kularen Oberflächenenergie bei binären äqui- molekularen Mischungen von Anilin und den drei isomeren Nitrophenolen
}

von

\author{
R. Kremann und E. Philippi. \\ Aus dem chemischen Institut der Universität Graz.
}

(Vorgelegt in der Sitzung am 9. Juli 1908.)

Vor einiger Zeit hatte der eine von uns gemeinsam mit R. Ehrlich ${ }^{1}$ in Übereinstimmung mit den Versuchen von W. Ramsay und Aston ${ }^{2}$ gezeigt, daß der Temperaturkoeffizient der molekularen Oberflächenenergie binärer äquimolekularer Mischungen sich additiv aus den Temperaturkoeffizienten der beiden Bestandteile zusammensetzt. Es zeigt derselbe den normalen Wert $2 \cdot 1$, wenn die beiden Stoffe $z u$ keinerlei Verbindung zusammentreten und man zur Berechnung des Wertes der molekularen Oberflächenenergie $v^{2 / s} \gamma$, als Molekularvolumen ( $\left.\frac{\text { Molekulargewicht }}{\text { Dichte }}\right) v$, das arithmetische Mittel der Molekularvolumina beider Komponenten einsetzt. Abweichungen vom normalen Werte können, wie wir an äquimolekularen Mischungen von Phenol-Anilin zeigten, ${ }^{3}$ dazu verwendet werden, nachzuweisen, ob und ungefähr bis zu welchem Grade zwei Stoffe im flüssigen Zustand zu Verbindungen zusammentreten, beziehungsweise fortbestehen.

1 Monatshefte für Chemie, 28, 831 (1907).

2 Zeitschr. für physik. Chemie, 15, 89 (1894).

3 Monatshefte für Chemie, 28, 831 (1907). 
Es lag nun ein gewisses Interesse vor, in dieser Hinsicht die binären äquimolekularen Systeme von Anilin und den drei isomeren Nitrophenolen zu untersuchen. Gemeinsam mit O. Rodinis ${ }^{1}$ hatte nämlich der eine von uns durch Aufnahme von Schmelzdiagrammen gezeigt, daß wohl $m$ - und $p$-Nitrophenol mit Anilin zu Verbindungen in äquimolekularem Verhältnis zusammentritt, dem $o$-Nitrophenol jedoch diese Fähigkeit nicht zukommt. Es war nun von Interesse festzustellen, ob $o$-Nitrophenol mit Anilin überhaupt zu einer Verbindung in nennenswertem Betrage zusammentritt oder ob die Verbindung lediglich in der Schmelze so weit dissoziiert, daß es nicht zur Ausscheidung einer eventuell existierenden Verbindung in festem Zustande, sondern nur der schwerer löslichen Komponente (o-Nitrophenol) kommt. Wir haben deshalb den Temperaturkoeffizienten der molekularen Oberflächenenergie sowohl von den drei isomeren Nitrophenolen als auch der äquimolekularen Mischungen derselben mit Anilin bestimmt. Von Anilin ist nach früheren Messungen bekannt, daß es den normalen Wert des Temperaturkoeffizienten der molekularen Oberflächenenergie zeigt.

Die Methode der Bestimmung der molekularen Oberflächenenergie war im wesentlichen die von Röntgen und Schneider ${ }^{2}$ angegebene.

Eine mit Milchglasskala versehene zylindrische Kapillare tauchte in ein sehr weites Proberohr ein. Letzteres enthielt die zu untersuchende Flüssigkeit, beziehungsweise Schmelze und befand sich in einem sehr großen Becherglas mit Wasser, das auf der gewünschten Temperatur erhalten wurde. Der Radius. der Kapillare wurde durch Auswägen mit Quecksilber ermittelt.

Die folgende Tabelle gibt die mit den drei isomeren Nitrophenolen sowie mit deren äquimolekularen Mischungen mit Anilin erhaltenen Resultate wieder. Als Temperaturkoeffizienten der molekularen Oberflächenenergie nahmen wir für Anilin $2 \cdot 1$ an.

1 Monatshefte für Chemie, 27, 125 (1906).

2 Wied. Ann., 29, 202 (1886). 
Tabelle.

\begin{tabular}{|c|c|c|c|c|c|c|c|c|c|c|}
\hline \multirow{2}{*}{\multicolumn{2}{|c|}{ 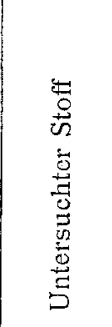 }} & \multirow{2}{*}{ 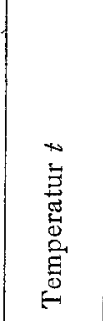 } & \multirow{2}{*}{ 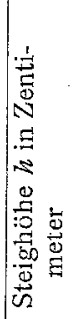 } & \multirow{2}{*}{ 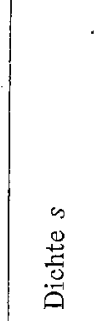 } & \multirow{2}{*}{ 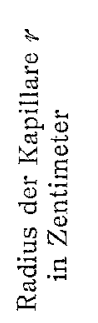 } & \multirow{2}{*}{ 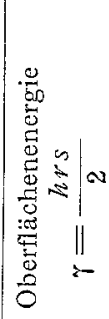 } & \multirow{2}{*}{ 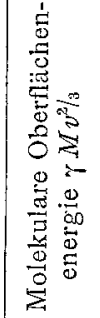 } & \multicolumn{3}{|c|}{$\begin{array}{l}\text { Temperatur- } \\
\text { koeffizient der } \\
\text { molekularen } \\
\text { Oberflächenenergie } \\
\frac{d\left[\gamma M v^{2} / 3\right]}{d t} \Delta\end{array}$} \\
\hline & & & & & & & & $\begin{array}{c}\Delta \\
\text { gef. }\end{array}$ & i. $\frac{\Delta}{\mathrm{M}}$ & $\begin{array}{c}\Delta \\
\text { ber. }\end{array}$ \\
\hline & 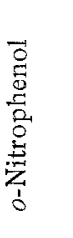 & $\begin{array}{l}55 \cdot 0^{\circ} \\
72 \cdot 0 \\
90 \cdot 5 \\
71 \cdot 0 \\
55 \cdot 5\end{array}$ & $\begin{array}{l}3 \cdot 47 \\
3 \cdot 38 \\
3 \cdot 29 \\
3 \cdot 40 \\
3 \cdot 48\end{array}$ & $\begin{array}{l}1 \cdot 2838 \\
1 \cdot 2656 \\
1 \cdot 2460 \\
1 \cdot 2666 \\
1 \cdot 2832\end{array} \mid$ & & $\begin{array}{l}40 \cdot 37 \\
38 \cdot 71 \\
36 \cdot 95 \\
38 \cdot 94 \\
40 \cdot 45\end{array}$ & $\begin{array}{l}917 \cdot 2 \\
888 \cdot 0 \\
856 \cdot 4 \\
892 \cdot 8 \\
919 \cdot 4\end{array}$ & $\begin{array}{l}1 \cdot 72 \\
1 \cdot 71 \\
1 \cdot 87 \\
1 \cdot 72\end{array}$ & $1 \cdot 75$ & - \\
\hline & 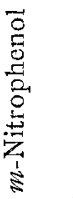 & $\begin{array}{l}115 \cdot 5^{\circ} \\
138 \cdot 5 \\
161 \cdot 5 \\
140 \cdot 0\end{array}$ & $\left|\begin{array}{l}3 \cdot 72 \\
3 \cdot 61 \\
3 \cdot 51 \\
3 \cdot 62\end{array}\right|$ & $\begin{array}{l}1 \cdot 256 \\
1 \cdot 228 \\
1 \cdot 200 \\
1 \cdot 225\end{array}$ & 0.0180 & $\begin{array}{l}42 \cdot 28 \\
40 \cdot 14 \\
38 \cdot 06 \\
40 \cdot 32\end{array}$ & $\begin{array}{c}974 \cdot 8 \\
939 \cdot 5 \\
904 \cdot 6 \\
941 \cdot 0\end{array}$ & $\left\{\begin{array}{l}1 \cdot 54 \\
1 \cdot 52 \\
1 \cdot 69\end{array}\right.$ & $1 \cdot 60$ & - \\
\hline & 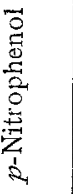 & $\begin{array}{c}131 \cdot 0^{\circ} \\
165 \cdot 0 \\
151 \cdot 0 \\
133 \cdot 0\end{array}$ & $\begin{array}{l}3 \cdot 90 \\
3 \cdot 69 \\
3 \cdot 76 \\
3 \cdot 84\end{array}$ & $\begin{array}{l}1 \cdot 327 \\
1 \cdot 259 \\
1 \cdot 289 \\
1 \cdot 323\end{array}$ & & $\begin{array}{l}46 \cdot 79 \\
42 \cdot 02 \\
43 \cdot 84 \\
46 \cdot 01\end{array}$ & $\begin{array}{r}1040 \cdot 0 \\
967 \cdot 6 \\
993 \cdot 5 \\
1027 \cdot 3\end{array}$ & $\begin{array}{l}2 \cdot 01 \\
1 \cdot 85 \\
1 \cdot 88\end{array}$ & 1.91 & 一 \\
\hline 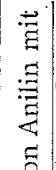 & $\begin{array}{cc}1 & \\
0 & 0 \\
\vdots & 0 \\
z & 0 \\
1 & 0 \\
0 & 0\end{array}$ & $\begin{array}{l}21 \cdot 0^{\circ} \\
42 \cdot 5 \\
65 \cdot 0\end{array}$ & $\begin{array}{l}4 \cdot 24 \\
4 \cdot 08 \\
3 \cdot 92\end{array} \mid$ & $\left(\begin{array}{l}1 \cdot 1784 \\
1 \cdot 1586 \\
1 \cdot 1380\end{array}\right)$ & & $\begin{array}{l}47 \cdot 19 \\
44 \cdot 64 \\
42 \cdot 13\end{array}$ & $\left|\begin{array}{c}1006.3 \\
962 \cdot 9 \\
919 \cdot 6\end{array}\right|$ & $\begin{array}{l}2 \cdot 02 \\
1 \cdot 93\end{array}$ & $1 \cdot 97$ & $1 \cdot 93$ \\
\hline 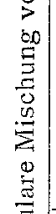 & 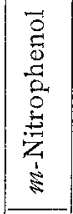 & $\begin{array}{l}27 \cdot 5^{\circ} \\
46 \cdot 0 \\
61 \cdot 0 \\
78 \cdot 0\end{array}$ & $\begin{array}{l}4 \cdot 46 \\
4 \cdot 30 \\
4 \cdot 16 \\
4 \cdot 04\end{array} \mid$ & $\begin{array}{c}1 \cdot 1946 \\
1 \cdot 1784 \\
1 \cdot 1656 \\
1 \cdot 1506\end{array} \mid$ & 0.018 & $\begin{array}{l}50 \cdot 31 \\
47 \cdot 86 \\
45 \cdot 79 \\
43 \cdot 90\end{array}$ & $\left|\begin{array}{r}1063 \cdot 4 \\
1020 \cdot 6 \\
983 \cdot 7 \\
951 \cdot 3\end{array}\right|$ & $\left\{\begin{array}{l}2 \cdot 31 \\
2 \cdot 46 \\
1 \cdot 91\end{array}\right.$ & $2 \cdot 23$ & $1 \cdot 85$ \\
\hline 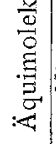 & 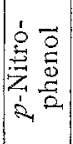 & $\begin{array}{l}41 \cdot 5^{\circ} \\
61 \cdot 2 \\
80 \cdot 0\end{array}$ & $\begin{array}{l}4 \cdot 18 \\
4 \cdot 06 \\
3 \cdot 94\end{array} \mid$ & $\begin{array}{l}1 \cdot 2022 \\
1 \cdot 1854 \\
1 \cdot 1690\end{array}$ & & $\begin{array}{l}48 \cdot 56 \\
45 \cdot 45 \\
43 \cdot 50\end{array}$ & $\begin{array}{r}1006 \cdot 4 \\
965 \cdot 5 \\
932 \cdot 6\end{array}$ & $\begin{array}{l}2 \cdot 08 \\
1 \cdot 75\end{array}$ & $1 \cdot 91$ & $2: 00$ \\
\hline
\end{tabular}


Man sieht aus den Versuchen, daß, wenn auch nur in geringem Maße, die drei Nitrophenole Tendenz zur Assoziation zeigen. Am stärksten ist dies noch bei dem $m$-Nitrophenol ausgeprägt. Die Temperaturkoeffizienten der äquimolekularen Mischungen geben nahezu ganz normale Werte. Wir sehen, daß $o$-Nitrophenol mit Anilin auch im flüssigen Zustand zu keiner Verbindung, wenigstens nicht in beobachtbarem Maße, zusammentritt. Der Umstand, daß auch die äquimolekularen Mischungen von $m$ - und $p$-Nitrophenol mit Anilin normale Werte des Temperaturkoeffizienten der molekularen Oberflächenenergie geben, trotzdem beide Stoffe mit Anilin Verbindungen im festen Zustand liefern, darf nicht befremden. Denn wir befinden uns bei der äquimolekularen Mischung in Konzentrations- und Temperaturintervallen, die bereits über den Umwandlungspunkten der betreffenden Verbindungen, d. i. bẹi $22 \cdot 5$, beziehungsweise $41 \cdot 5$ liegen.

Auffällig ist besonders bei der äquimolekularen Mischung. $m$-Nitrophenol-Anilin, daß wir normale Werte des Temperaturkoeffizienten der molekularen Oberflächenenergie beobachten, obschon der aus den Werten derselben für die beiden Komponenten berechnete Mittelwert kleiner ist als der normale Wert. Wir können dies jedoch einfach dadurch erklären, daß in der äquimolekularen Mischung das Assoziationsausgleichsgewicht des betreffenden Nitrophenols infolge der Volumvermehrung zugunsten einfacher Molekel verschoben wird, indem ja im System beim Übergang vom reinen Nitrophenol zur äquimolekularen Mischung die Konzentration des Nitrophenols von 1.0 auf 0.5 sinkt. 